www. revistadyo.com

\title{
Atención al gap entre RSE percibida y esperada por los empleados: una nueva aproxi- mación estratégica
}

Dr. Alejandro Alvarado Herrera

Recibido: 14 de Julio de 2018 / Aceptado: 12 de Noviembre de 2018

\section{Resumen}

Para mejorar la toma de decisiones estratégicas y desarrollar iniciativas y programas de responsabilidad social empresarial (RSE) más efectivos y eficientes, es necesario contar con instrumentos de análisis más poderosos y precisos que los conocidos hasta hoy. Con el objetivo de concebir, desarrollar y contrastar un modelo explicativo de los gaps o brechas entre RSE percibida y esperada (RSEp-e) y sus influencias sobre el comportamiento de los empleados, se realizó una investigación ad-hoc, cuyos resultados permiten introducir en la literatura: la noción de gap RSEp-e y el modelo en cuestión. Se abordan también las principales implicaciones académicas y gerenciales.

\section{Palabras clave}

Responsabilidad Social Corporativa; Disconfirmación de Expectativas; Gap RSE percibida-esperada; Comportamiento de los empleados; Modelos de Ecuaciones Estructurales

\section{Introducción}

La responsabilidad social empresarial (RSE) ha sido identificada en la literatura como un imperativo estratégico para las organizaciones que tratan de desarrollar ventajas competitivas sostenibles en el mercado (Serra, Peña, Ramón y Martorell, 2017). En efecto, la RSE ocupa un lugar prominente en la agenda de negocios actual dada su capacidad para dar respuesta a las preocupaciones sociales y medioamientales de sus stakeholders procurando satisfacer sus expectativas mediante la creación de valor compartido (Janssen, Sen y Bhattacharya, 2015; Martínez y Rodríguez del Bosque, 2016).

Efectivamente, las compañías alrededor del mundo dedican actualmente mayores esfuerzos y recursos que nunca antes a sus programas e iniciativas de RSE (Serra et al., 2017) por lo que resulta explicable que la atención académica y profesional sobre el tema se haya acrecentado de forma notable a lo largo de las últimas décadas. De hecho, los estudios centrados en la RSE, su relaciones y efectos sobre el comportamiento de sus consumidores, empleados, inversionistas, proveedores y comunidades en las que operan las firmas han crecido en cantidad y evolucionado en términos epistemológicos (Bigné, Alvarado, Currás y Rivera, 2010; Serra et

\footnotetext{
Dr. Alejandro Alvarado Herrera * alex.alvarado.dr@tec.mx

*Departamento de Mercadotecnia y Análisis. Escuela de Negocios. Tecnológico de Monterrey Campus Sonora Norte +52 662 259-1000 https://orcid.org/0000-0002-9902-6766
}

al., 2017). Esto ha propiciado el descubrimiento de lagunas en el conocimiento y evidenciado la necesidad de desarrollar nuevas líneas de estudio sobre la RSE, sus antecedentes y consecuentes. El presente trabajo se suma a esas investigaciones y pretende contribuir a llenar de forma primigenia algunos de esos vacíos y necesidades como sigue.

En primer lugar, la literatura señala la necesidad de contar con modelos y explicaciones más completas y poderosas que las actuales acerca de la RSE (Mejías, García, Prado, Fernández y Comesaña, 2011) y de sus influencias sobre variables clave del comportamiento de los stakeholders (Swaen, 2003). Modelos que redunden en mejoras en la toma de decisiones directivas y que permitan a las empresas alinear adeuadamente sus planteamientos estratégicos, de gestión y operativos con la RSE (Mejías et al., 2011).

El segundo vacío del conocimiento identificado en la literatura, y que pretende ser atendido en este trabajo, se deriva de la todavía relativamente escasa investigación de las influencias de la RSE sobre el comportamiento de los empleados. Lo anterior, aún cuando se está al tanto acerca de la preocupación y el compromiso de los stakeholders internos por el medioambiente (Vintró y Fortuny, 2010) y de que la RSE influye en el bienestar, compromiso, motivación e identificación de los empleados con la organización; factores determinantes para la calidad de la vida laboral y, consecuentemente, para la mejora de los productos y servicios de la organización, la satisfacción de sus consumidores y la competitividad de la firma (Kim, Song, Lee y Lee, 2017; Serra et al., 2017). Esta necesidad es especialmente notoria en el caso de las empresas prestadoras de servicios, dada la inseparabilidad de los recursos humanos y la entrega del producto (Bañuls, Rodríguez y Jiménez, 2007; Organización Internacional del Trabajo, 2001; Serra et al., 2017). 
Finalmente, la tercera laguna hallada en la literatura surge de evidencia empírica que señala que los postulados y la capacidad explicativa de los modelos teóricos que han predominado en el estudio de las percepciones y opiniones de los stakeholders sobre la RSE, a saber: la pirámide de la responsabilidad social corporativa (Carroll, 1979 y 1999) y las asociaciones corporativas (AC) (Brown y Dacin, 1997), tienen limitaciones significativas (García de los Salmones, Herrero y Rodríguez del Bosque, 2005; Alvarado y Schlesinger, 2008; Alvarado, Bigné, Aldás y Currás, 2017) y no necesariamente son válidos o aplicables para contextos distintos al mercado de bienes de consumo masivo estadounidense (Maignan, 2001; Maignan y Ferrell 2003).

Esta investigación pretende dar continuidad a los avances científicos logrados hasta ahora y contribuir a llenar, inicial y parcialmente, estos tres vacíos mediante la generación de conocimientos nuevos surgidos del estudio de los gaps o brechas entre las percepciones y las expectativas de los empleados de empresas de servicios acerca de la RSE, y sus influencias en variables consecuentes que son relevantes para su desempeño: identificación organizacional (IO), motivación laboral (ML) e intención de permanencia en la firma (IP).

Atendiendo a estas oportunidades, se diseñó y llevó a cabo una investigación ad-hoc cuantitativa, de sección transversal y naturaleza confirmatoria con el triple objetivo de: i) estudiar los gaps entre las percepciones y las expectativas de los empleados de empresas de servicios acerca de la RSE, ii) conocer sus influencias sobre su IO, ML e IP; y iii) validar o refutar externamente la idoneidad del modelo de las AC para medir y explicar con precisión fenómenos de la RSE.

\section{Antecedentes de la investigación}

\subsection{RSE, stakeholders y el paradigma de la disconfirmación de expectativas}

Si bien es posible hallar indicios y evidencias antiguos del interés de la comunidad empresarial sobre aspectos sociales de sus actividades (Brønn y Vrioni, 2001), se considera que el inicio del debate sobre la naturaleza de las responsabilidades empresariales y el estudio de la RSE tuvo lugar con la publicación del libro Social Responsibilities of the Businessman (Bowen, 1953). Unos años después, y en respuesta a la perspectiva de la primacía de los accionistas (shareholders) (Friedman, 1962), apareció el concepto de los grupos de interés o stakeholders; término "atribuido a un grupo de académicos del Stanford Research Institute" (Stoney y Winstanley, 2001) quienes propusieron que las empresas, además de enfocarse en los accionistas, han de: "responsabilizarse de una variedad de stakeholders sin cuyo soporte la organización dejaría de existir" (Ídem, p.604). Posteriormente, Freeman y Reed dieron un sentido más amplio al término, definiéndolo como: cualquier grupo o individuo identificable que pueda afectar o ser afectado por el logro de los objetivos de la organización (1983), dando paso, a partir de los postulados de la teoría organizativa de Barnard (1938), al desarrollo de la teoría de los stakeholders.

La visión de esta teoría, fundamentalmente estratégica, proclama que la RSE es capaz de potenciar la ventaja competitiva de las empresas (Heugens, van den Bosch y van Riel, 2002), ya que supone que la implicación de la empresa en determinadas actividades de RSE que los stakeholders perciben como importantes para ellos puede ser beneficiosa para la misma, y la no implicación puede llevarlos a retirarle su apoyo a la organización (McWilliams, Siegel y Wright, 2006), por ello, ha servido como base para numerosos estudios en el ámbito de la RSE, tal como es el caso del presente trabajo.

Por otra parte, es interesante notar que aún cuando las organizaciones están enfocadas en satisfacer las expectativas de sus stakeholders, dadas las importantes oportunidades de negocio que ello supone (Luque-González, Hernández-Zubizarreta y de Pablos-Heredro, 2016), una parte importante de las investigaciones de RSE centradas en el estudio del comportamiento de los miembros de los grupos de interés se basan en sus percepciones sobre los programas, acciones e iniciativas llevadas a cabo por las firmas, obviando, precisamente sus expectativas. Ejemplos de esos trabajos valiosos pueden encontrarse en distintos sectores y contextos temporales, geográficos y culturales (v.g. Gorden, Anderson y Bruning, 1992; Kim et al., 2017; Markovic, Iglesias, Singh y Sierra, 2018; Serra et al., 2017; Vintró y Fortuny, 2010).

Ahora bien, tal como establece Swaen (2003), el análisis integral de los gaps entre las percepciones y las expectativas de los individuos en relación al compromiso social de la compañía puede constituir una base sólida para lograr explicaciones más poderosas que las actuales sobre la RSE y sus consecuentes actitudinales y comportamentales. Luego, el paradigma de la disconfirmación de expectativas (PDE) (Oliver, 1980) constituye un marco adecuado para acometer la comparación entre las expectativas de los stakeholders acerca de las actividades e iniciativas de responsabilidad social de las empresas y lo que perciben que éstas hacen al respecto.

A lo largo del tiempo, el PDE ha sido ampliamente utilizado en la literatura de marketing y del comportamiento del consumidor (Bhattacherjee, 2001) para estudiar causas y efectos cognitivos de la satisfacción (Anderson y Sullivan, 1993; Oliver, 1980), la calidad y experiencia en el servicio (Parasuraman, Zeithaml y Berry, 1985) y las intenciones de comportamiento (Cronin, Brady y Hult, 2000), entre otras. Sus postulados han sido también incorporados ya a otros ámbitos y grupos de estudio, tales como el del comportamiento de los usuarios de sistemas de información (Bhattacherjee, 2001) y el del comportamiento de los empleados y su calidad de vida laboral (Kandasamy y Ancheri, 2009), e in- 
clusive el comportamiento de los consumidores de servicios turísticos (Alvarado, Bigné, Currás y Aldás, 2010).

En este último trabajo referido, Alvarado et al. (2010) utilizaron el PDE de forma análoga a la tradicionalmente empleada pero en relación a la RSE. Es decir, conceptualizando y operacionalizando los conceptos RSE percibida (RSEp), RSE esperada (RSEe) y el gap entre ambas nociones (Gap RSEp-e), bajo la premisa de Swaen (2003) de que, mientras mayor sea la RSEp en comparación con la RSEe, los sujetos, presumiblemente, tenderán a reaccionar más favorablemente hacia la empresa. Luego, para los fines de esta investigación, y adaptando las mismas definiciones a los empleados, se entiende por RSEe: los deseos o anhelos de los empleados respecto a las actividades de RSE de la firma para la que prestan sus servicios; por RSEp: los juicios de los empleados acerca del desempeño de la organización respecto a las actividades de RSE llevadas a cabo por la propia empresa; y por Gap RSEp-e: la discrepancia entre ambos conceptos.

El documento referido es, hasta donde llega nuestro conocimiento, el primer avance formal en la investigación sobre el estudio sobre el uso del PDE en el ámbito de la RSE, por lo que constituye la principal base conceptual y operacional de este trabajo. Sus postulados y hallazgos requieren, por consiguiente, de contrastación y validación externa adicionales pero centradas en grupos de interés, realidades y contextos distintos a los de su origen, al tiempo que ha de procurarse la comparabilidad de sus resultados en la medida de lo posible. Consecuentemente, el subsector de servicios de alojamiento turístico, la metodología y los instrumentos de medición y análisis ahí descritos son los mismos que los utilizados para el desarrollo de este trabajo. Sin embargo, el grupo de stakeholders, las variables consecuentes del Gap RSEp-e y el contexto geográfico aquí estudiados son distintos.

\subsection{IO, ML e IP como variables consecuentes del Gap RSEp-e}

Por otro lado, como se anticipó, la literatura señala que la RSE puede influir en distintas variables del comportamiento de los trabajadores. La primera variable consecuente del Gap RSEp-e considerada en esta investigación es la IO, la cual, de acuerdo a Mael y Ashforth (1992), es un tipo concreto de identificación social, en la que el grupo o categoría social donde se ubica el sujeto es una organización. Se considera que la IO es capaz de dotar a los individuos de sentido de pertenencia y conexión con la empresa; "la pertenencia a una organización de trabajo es una de las afiliaciones sociales más importantes que una persona posee, porque ocupa más tiempo vital del que es gastado en otros grupos, y porque el sustento de la misma depende de la fortuna de la organización” (Bergami y Bagozzi, 2000, p. 555).

Producto de este sentido de conexión, la organización es psicológicamente aceptada como parte de la identidad personal (Scott y Lane, 2000), convirtiendo a la IO en una variable capaz de desarrollar conductas deseables de los empleados, potenciar su rendimiento y el de la firma, en la medida que éstos participan y adoptan los objetivos de la empresa. Cabe esperar entonces que:

$H_{1}$ : El Gap RSEp-e in luye directa, positiva y signi icativamente en la IO de los empleados de la firma.

Por su parte, la IO basada en la RSE debe influir en el compromiso afectivo del empleado con la empresa (Brammer, Millington y Rayton, 2007; Kim, Lee, Lee y Kim, 2010; Serra et al., 2017) ya que, al identificarse con una empresa socialmente responsable, los empleados pueden apropiarse de la personalidad de la organización para proyectar la propia, satisfaciendo con ello sus necesidades de definición personal (Bhattacharya, Korschun y Sen, 2008; Kim et al., 2010). Así, el mayor sentido de identificación con la firma, derivado de las percepciones y expectativas de RSE se traducirá en un mayor impulso motivacional hacia el trabajo, pues los objetivos organizacionales pasan a ser, de algún modo, también objetivos personales (Dutton, Dukerich y Harquail, 1994; Bhattacharya y Sen, 2003). Por lo tanto, cabe esperar que:

$\mathrm{H}_{2}$ : La IO basada en la RSE in luye directa, positiva y significativamente en la ML de los empleados de la empresa.

La literatura también indica que los sujetos desean mantener un cierto sentido de coherencia y continuidad en su autoconcepto (Dutton et al., 1994). Por ello, los empleados identificados con una empresa socialmente responsable estarán dispuestos a mantener su relación laboral en el largo plazo, lo que supone esperar de ellos una mayor intención de permanencia en la organización, es decir:

$H_{3}$ : La IO basada en la RSE in luye directa, positiva y significativamente en la IP de los empleados en la compañia.

Por último, se conoce también que la motivación hacia el trabajo es, a su vez, un factor precursor de la permanencia de los empleados ya que, como puede desprenderse de los postulados de Sager, Naletelich y Dubinsky (2014) al respecto, aquellos que perciben su lugar de trabajo como apto para el desarrollo de sus habilidades positivas y su empleo como satisfactorio, desean permanecer mayor tiempo en la organización. Luego es previsible que:

$H_{4}: \quad$ La ML del empleado in luye directa, positiva y significativamente en su IP.

En la Figura 1 se presenta el modelo teórico resultante de la incorporación de estas cuatro hipótesis de las influencias directas e indirectas del Gap RSEp-e sobre la IO, la ML y la IP de los empleados. 
Figura 1 Modelo teórico propuesto

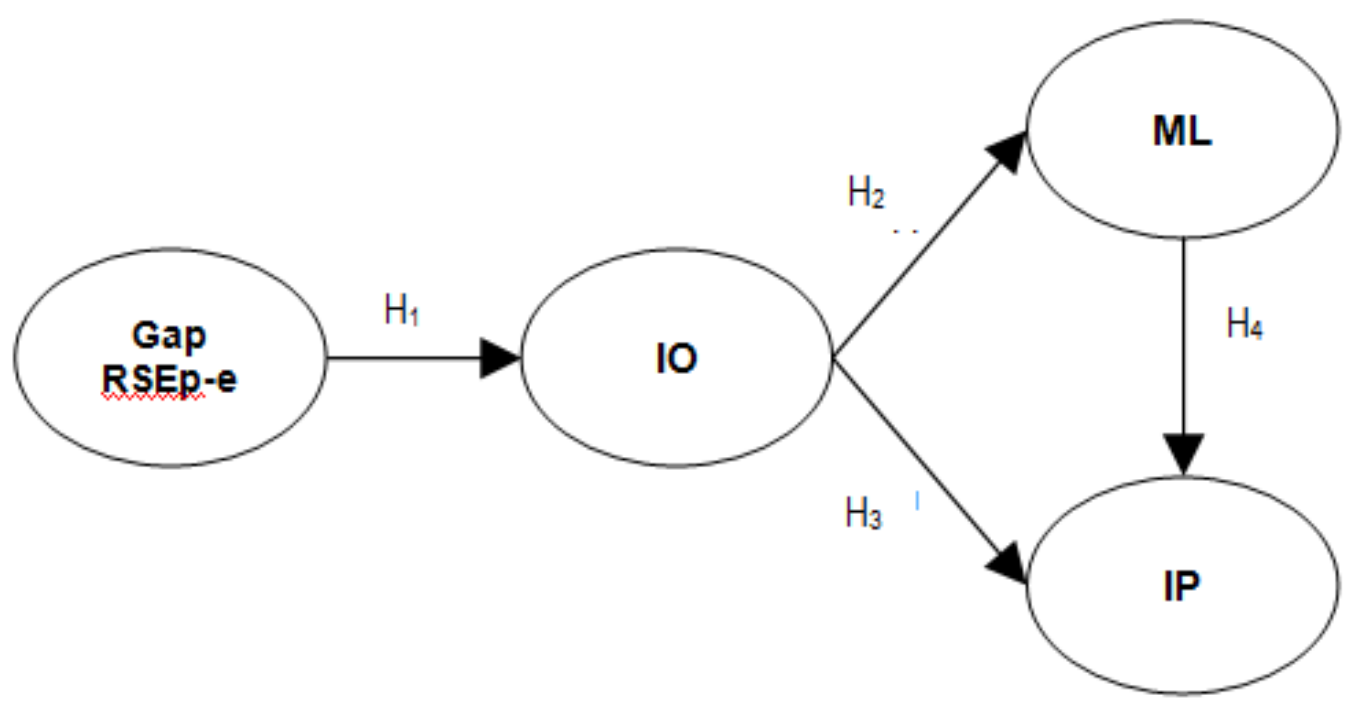

\section{Metodología de la investigación}

La contrastación empírica del modelo propuesto se realizó mediante investigación cuantitativa, confirmatoria y de sección transversal. Las observaciones se hicieron con un cuestionario estructurado auto-administrado y la estimación de sus relaciones estructurales mediante el análisis de sus estructuras de varianzas y covarianzas con EQS 6.3 (Bentler 1985-2017).

Siguiendo las recomendaciones de Serra et al. (2017) y Alvarado et al. (2010), se optó por aplicar el estudio en el sector turístico, específicamente en servicios de alojamiento, dado que sus características confieren a ese subsector cierta unicidad que resulta propicia para el estudio de la RSE, a saber: i) las características de sus recursos humanos y de su gestión (Bañuls et al., 2007; OIT, 2001); ii) sus impactos en las comunidades en las que operan; iii) la intensa competencia (Bañuls et al., 2007; Serra et al., 2017); iv) se trata de un ámbito no estigmatizado del que no se esperan efectos adversos apriorísticos por falta de legitimidad social (Dean 2002); v) la información puede ser recabada en condiciones reales; vi) se consideran razonablemente satisfechas las condiciones evolutivas (McEnally y De Chernatony 1999) y de homogeneidad de las marcas (Barone et al., 2000) y producto (Mohr y Webb 2005); y vii) permite ampliar y validar externamente, en su caso, los hallazgos del estudio base de esta investigación.

La recolección de los datos se llevó a cabo en tres cadenas hoteleras de reconocido prestigio y calidad en la isla de Cozumel, México; destino turístico internacional consolidado que cuenta con 4098 habitaciones en 45 hoteles (Instituto Nacional de Estadística y Geografía [INEGI], 2016). La población estudiada fue la correspondiente a los empleados de las distintas áreas y niveles jerárquicos de las empresas seleccionadas; su participación fue voluntaria y anónima. El tamaño de la muestra se determinó atendiendo a las sugerencias de Kline (2011) y Küster, Vila y Aldás (2000) en el sentido de que ésta: i) debe ser lo suficientemente grande como para asegurar que la varianza producida por un solo individuo sea despreciable y ii) que el mínimo recomendable para contrastar modelos de ecuaciones estructurales y lograr cierta generalización de los resultados es de 200 observaciones, se decidió fundamentar esta investigación en una muestra total de 205 empleados, los cuales fueron seleccionados aleatoriamente en sus centros de trabajo.

El cuestionario fue diseñado ex profeso, de conformidad con el procedimiento y los criterios recomendados por Malhotra (2008) e incluyó cinco partes destinadas a medir RSEp, RSEe, IO, ML e IP respectivamente; se utilizaron las escalas compuestas descritas más adelante, empleando un formato tipo Likert con 7 puntos de asignación de respuesta anclados en "totalmente en desacuerdo" y "totalmente de acuerdo". Es importante señalar que las escalas seleccionadas han demostrado previamente contar con fiabilidades simples y compuestas superiores a los valores críticos mínimos aceptables de $\propto^{\prime} \geq 0.7$ e IFC' $\geq 0.7$ (Nunally y Bernstein, 1994). La última parte del cuestionario se destinó a la obtención de datos sociodemográficos de los participantes.

Así, para la medición del Gap RSEp-e se emplearon los mismos seis ítems que los del estudio base de este trabajo, a saber: los tres originales del modelo de las AC (Brown y Dacin, 1997) para medir la RSEp (RSEp $\left.1-\mathrm{RSEp}_{3}\right)$ y los tres complementarios requeridos para su contraparte RSEe $\left(\mathrm{RSEe}_{1}-\mathrm{RSEe}_{3}\right)$ siguiendo la adaptación de Alvarado et al. (2010). En lo referente a las variables consecuentes se emplearon: los siete ítems propuestos por Escalas y Bettman (2003) para medir la $\mathrm{IO}\left(\mathrm{IO}_{1}-\mathrm{IO}_{7}\right)$; cuatro ítems para la $\mathrm{ML}$ $\left(\mathrm{ML}_{1}-\mathrm{ML}_{4}\right)$ y tres ítems para la medición de la IP $\left(\mathrm{IP}_{1}-\mathrm{IP}_{3}\right)$ adaptados de Chandon, Morwitz y Reinartz (2005). El contenido de cada uno de los reactivos aquí descritos se muestra en el Anexo 1 de este documento. 


\section{Análisis y discusión de los resultados}

En esta sección se analizan y discuten los principales hallazgos de la investigación en cinco apartados. En primer lugar se tratan los estadísticos descriptivos correspondientes al perfil de los participantes, seguido de los análisis de la fiabilidad y validación del modelo de medida. Posteriormente se abordan los descriptivos referentes a la RSEp, RSEe, los gaps RSEp-e y las variables consecuentes, para dar paso a la contrastación de las hipótesis y del modelo en su conjunto.

\subsection{Perfil de los participantes}

Las características de los participantes se presentan en la Tabla 1.

Tabla 1 Perfil de los participantes

\begin{tabular}{|c|c|c|c|}
\hline \multirow[t]{2}{*}{ Variable } & \multirow[t]{2}{*}{ Definición } & \multicolumn{2}{|c|}{ Frecuencia } \\
\hline & & Absoluta & $(\%)$ \\
\hline \multirow[t]{2}{*}{ Sexo } & Masculino & 178 & 74.0 \\
\hline & Femenino & 52 & 26.0 \\
\hline \multirow[t]{8}{*}{ Edad } & 18 años o menos & 19 & 9.5 \\
\hline & De 19 a 28 años & 74 & 37.0 \\
\hline & De 29 a 38 años & 46 & 23.0 \\
\hline & De 39 a 48 años & 32 & 16.0 \\
\hline & De 49 a 58 años & 16 & 8.0 \\
\hline & De 59 a 68 años & 10 & 5.0 \\
\hline & Más de 68 años & 1 & 0.5 \\
\hline & No sabe / No contesta & 2 & 1.0 \\
\hline \multirow[t]{11}{*}{ Área de trabajo } & Alimentos y Bebidas & 79 & 39.5 \\
\hline & División cuartos & 33 & 16.5 \\
\hline & Mantenimiento & 17 & 8.5 \\
\hline & Seguridad & 16 & 8.0 \\
\hline & Áreas públicas & 14 & 7.0 \\
\hline & Otros & 12 & 6.0 \\
\hline & Contabilidad & 11 & 5.5 \\
\hline & Recepción & 5 & 2.5 \\
\hline & Animación & 5 & 2.5 \\
\hline & Recursos Humanos & 4 & 2.0 \\
\hline & Ventas & 3 & 1.5 \\
\hline
\end{tabular}




\begin{tabular}{|c|c|c|c|}
\hline & No sabe / No contesta & 1 & 0.5 \\
\hline \multirow[t]{7}{*}{ Puesto de trabajo } & Empleado de línea & 152 & 76.0 \\
\hline & Supervisor & 19 & 9.5 \\
\hline & Encargado de área & 14 & 7.0 \\
\hline & Asistente & 7 & 3.5 \\
\hline & Gerente & 5 & 2.5 \\
\hline & Jefe & 2 & 1.0 \\
\hline & No sabe / No contesta & 1 & 0.5 \\
\hline \multirow[t]{3}{*}{ Hotel } & Uno & 70 & 35.0 \\
\hline & Dos & 29 & 14.5 \\
\hline & Tres & 101 & 50.5 \\
\hline
\end{tabular}

Como puede apreciarse: $74 \%$ fueron hombres y $26 \%$ mujeres. El rango de edades de mayor presencia fue el que comprende de 19 a 28 años (37\%), seguido del comprendido entre 29 y 38 años (16\%), mientras que únicamente 5.5\% de los trabajadores que respondieron el cuestionario dijeron tener 59 años o más. En lo que a las áreas de trabajo se refiere, $39.5 \%$ de quienes respondieron dijeron estar adscritos a Alimentos y bebidas, grupo seguido en número por los miembros de la División de cuartos (16.5\%); en tanto que, la mayor parte de los participantes refirieron ser empleados de línea (76\%) y $9.5 \%$ supervisores. Los datos sugieren que la muestra obtenida es razonablemente adecuada para su estudio, pues aún cuando se observa una participación de mujeres menor a la esperada, los empleados del sector turístico suelen ser en su mayoría jóvenes y ocupar puestos operativos tales como recepcionistas, mozos, botones, camareros, cocineros, etcétera (OIT, 2001).

\subsection{Fiabilidad y validación del modelo de me- dida}

Con los datos recabados se llevó a cabo un análisis factorial confirmatorio (AFC), el cual fue estimado con EQS 6.3 (Bentler 1985-2017) usando el método de máxima verosimilitud y empleando estadísticos robustos (Satorra y Bentler, 1988) para corregir su no normalidad (Coeficiente de Mardia
Normalizado Estimado=57.18). Los resultados sugirieron la conveniencia de desechar cinco casos, dadas sus altas contribuciones al sesgo en las respuestas por lo que, luego de eliminarlos, se llevó a cabo un segundo AFC con los 200 casos retenidos, cuyos resultados se muestran en la Tabla 2.

Como se desprende de su lectura, los resultados su-gieren un buen ajuste del modelo de medida (S-B $\times 2$ $(113 \mathrm{gl})=156.88, \quad \mathrm{p}<0.01 ; \quad \mathrm{NFI}=0.886 ; \quad \mathrm{NNFI}=0.956$; $\mathrm{CFI}=0.964 ; \mathrm{IFI}=0.964 ; \mathrm{MFI}=0.892$ y $\mathrm{RMSEA}=0.05)$, lo que supone un primer indicio de validez convergente. Otros aspectos que apuntan también a la consistencia interna del modelo son que: i) las fiabilidades simples de cada factor resultan superiores al valor crítico de $\propto^{\prime} \geq 0.7$ (Nunnally y Bernstein, 1994); ii) todos los ítems son estadísticamente significativos $(\mathrm{p}<0.01)$ respecto a su factor; iii) las magnitudes y la dirección de sus cargas son superiores al valor crítico $\lambda^{\prime} \geq 0.6$ (Bagozzi y Yi, 1988); iv) los promedios de dichas cargas respecto a su factor también son superiores al valor crítico $\lambda^{\prime} \geq 0.7$ (Hair et al., 1998); v) los índices de fiabilidad compuesta correspondientes a cada factor son superiores al valor deseable IFC' $\geq 0.6$ (Bagozzi y Yi 1988) y vi) los valores obtenidos de los índices de varianza extraída sobrepasan el valor crítico de IVE' $\geq 0.50$ (Fornell y Larcker 1981). Visto que el modelo de medida satisface ampliamente con los requerimientos señalados, se considera suficientemente probada su validez convergente. 
Dr. Alejandro Alvarado Herrera. / Dirección y Organización 67 (2019) 5-21

Tabla 2 Resultados del AFC y propiedades psicométricas del modelo de medida

\begin{tabular}{ccccccc}
\hline Factor & \multicolumn{3}{c}{ Fiabilidad } & \multicolumn{2}{c}{ Validez convergente } \\
\cline { 2 - 6 } & $\propto$ & IFC & IVE & $\lambda$ estandarizada & $\lambda$ promedio
\end{tabular}

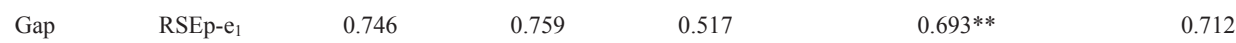

RSEp-e
RSEp-e ${ }_{2}$
0.840 **

RSEp-e $_{3}$

$0.604 * *$

\begin{tabular}{|c|c|c|c|c|c|c|}
\hline & RSEp-e & & & & $0.604 * *$ & \\
\hline \multirow[t]{7}{*}{ IO } & $\mathrm{IO}_{1}$ & 0.903 & 0.910 & 0.593 & $0.642 * *$ & 0.767 \\
\hline & $\mathrm{IO}_{2}$ & & & & $0.714^{* *}$ & \\
\hline & $\mathrm{IO}_{3}$ & & & & $0.879 * *$ & \\
\hline & $\mathrm{IO}_{4}$ & & & & $0.791^{* *}$ & \\
\hline & $\mathrm{IO}_{5}$ & & & & $0.777 * *$ & \\
\hline & $\mathrm{IO}_{6}$ & & & & $0.775 * *$ & \\
\hline & $\mathrm{IO}_{7}$ & & & & $0.790 * *$ & \\
\hline \multirow[t]{4}{*}{ ML } & $\mathrm{ML}_{1}$ & 0.935 & 0.935 & 0.783 & $0.883^{* *}$ & 0.884 \\
\hline & $\mathrm{ML}_{2}$ & & & & $0.836^{* *}$ & \\
\hline & $\mathrm{ML}_{3}$ & & & & $0.947 * *$ & \\
\hline & $\mathrm{ML}_{4}$ & & & & $0.801 * *$ & \\
\hline \multirow[t]{3}{*}{ IP } & $\mathrm{IP}_{1}$ & 0.777 & 0.858 & 0.669 & $0.652 * *$ & 0.817 \\
\hline & $\mathrm{IP}_{2}$ & & & & $0.782 * *$ & \\
\hline & $\mathrm{IP}_{3}$ & & & & $0.787 * *$ & \\
\hline
\end{tabular}

Indicadores de bondad de ajuste

\begin{tabular}{ccccccc}
\hline & & & & & \\
S-B $\chi^{2}(113 \mathrm{gl})=156.88^{* *}$ & NFI & NNFI & CFI & IFI & MFI & RMSEA \\
\cline { 2 - 6 } & & & & & & \\
& 0.886 & 0.956 & 0.964 & 0.964 & 0.892 & 0.05 \\
\hline
\end{tabular}

** $\mathrm{p}<0.01$ 
En la Tabla 3 se presentan los datos necesarios para la realización de dos pruebas convencionales para el establecimiento de la validez discriminante del modelo de medida: i) test de los intervalos de confianza (Anderson y Gerbing, 1988) y ii) test de la varianza extraída (Fornell y Larcker 1981). Como se aprecia, el modelo cumple con los requerim- ientos correspondientes, a saber: i) ninguno de los intervalos de confianza comprendidos entre $\pm 2 \sigma$ de las estimaciones resultantes (95\%) incluye el valor de $|1.0|$, y ii) los IVE de cada factor superan, en todos los casos, los cuadrados de las correlaciones de cada par de factores por lo que se concluye que el modelo de medida posee validez discriminante.

Tabla 3 Validez discriminante: IVE, intervalos de confianza y corr2

\begin{tabular}{ccccc}
\hline Factor & Gap RSEp-e & IO & ML & IP \\
\hline Gap RSEp-e & 0.517 & {$[0.19,0.51]$} & {$[0.213,0.541]$} & {$[0.035,0.423]$} \\
\hline IO & 0.123 & & & \\
ML & 0.142 & 0.593 & {$[0.555,0.875]$} & {$[0.634,0.870]$} \\
\hline IP & & & & 0.783 \\
\hline
\end{tabular}

Nota: En la diagonal se presentan los IVE, arriba de la diagonal los intervalos de confianza para cada par de factores y bajo la diagonal los cuadrados de los coeficientes de correlación.

\subsection{Estadísticos descriptivos de las variables estudiadas}

\subsubsection{RSEp, RSEp y Gap RSEp-e}

Para medir la disconfirmación de las percepciones y las expectativas de los empleados en torno a la RSE de la firma para la que trabajan, fueron usados los tres ítems de la escala de Brown y Dacin (1997) para la RSEp antes referidos y, los mismos tres reactivos pero con los ajustes necesarios en sus enunciados para enfocar la RSEe. Luego, se calculó la diferencia entre ambas para determinar el Gap RSEp-e para cada observación. Los valores medios resultantes se presentan en la Tabla 4 a continuación.
Tabla 4 Estadísticos descriptivos de la RSE

\begin{tabular}{|c|c|c|c|c|c|}
\hline Variable & Ítem & $\bar{x}$ & $\sigma$ & Min. & Max \\
\hline \multirow[t]{3}{*}{ RSEp } & $\mathrm{RSEp}_{1}$ & 5.06 & 1.85 & 1 & 7 \\
\hline & $\mathrm{RSEp}_{2}$ & 4.87 & 1.91 & 1 & 7 \\
\hline & $\mathrm{RSEp}_{3}$ & 5.66 & 1.55 & 1 & 7 \\
\hline \multirow[t]{3}{*}{ RSEe } & $\mathrm{RSEe}_{1}$ & 6.05 & 1.28 & 1 & 7 \\
\hline & $\mathrm{RSEe}_{2}$ & 6.07 & 1.20 & 1 & 7 \\
\hline & $\mathrm{RSEe}_{3}$ & 6.74 & 0.66 & 4 & 7 \\
\hline \multirow[t]{3}{*}{ Gap RSEp-e } & Gap RSEp-e ${ }_{1}$ & -0.97 & 2.00 & -6 & 7 \\
\hline & Gap RSEp-e ${ }_{2}$ & -1.21 & 2.10 & -6 & 4 \\
\hline & Gap RSEp-e $_{3}$ & -1.11 & 1.55 & -6 & 2 \\
\hline
\end{tabular}


Como puede observarse, los valores medios de las percepciones de los empleados sobre las iniciativas de RSE emprendidas por sus empresas resultaron favorables en cada caso, siendo el aspecto mejor valorado el correspondiente a sus esfuerzos en favor de la protección al medio ambiente $\left(\bar{x} \mathrm{REp}_{3}=5.66\right)$, seguido por los referentes a sus donaciones económicas a causas sociales $\left(\overline{\mathrm{xSEp}} \mathrm{R}_{1}=5.06\right) \mathrm{y}$, en menor magnitud, los vinculados a su contribución al mejoramiento en la calidad de vida de la comunidad local $\left(\bar{x} \mathrm{RSEp}_{2}=4.87\right)$. En tanto que las mayores expectativas de estos stakeholders correspondieron también a su interés por cuidar el medio ambiente ( $\left.\bar{x} \mathrm{RSEe}_{3}=6.74\right)$, seguidas de forma prácticamente indistinta por las relacionadas con la necesidad de mejorar la calidad de vida de su comunidad $\left(\bar{x} \mathrm{RSEe}_{2}=6.07\right)$ y por hacer donaciones económicas $\left(\bar{x} \mathrm{RSEe}_{1}=6.05\right)$ respectivamente.

Como resultado de que los valores medios adoptados por los ítems referentes a las percepciones hayan sido, en todos los casos, inferiores a las expectativas, se tienen sendas disconfirmaciones negativas. El sentido negativo de estos gaps significa que, aún cuando los empleados percibieron positivamente los esfuerzos de RSE realizados por sus empresas, las cadenas hoteleras para las que trabajan fueron incapaces de satisfacerlos plenamente en ninguno de los aspectos estudiados. La mayor de esas brechas es la que atañe a la mejora de la condiciones de vida de su comunidad ( $\bar{x}$ GapRSEp- $\mathrm{e}_{2}=-1.21$ ), seguida por la concerniente a los aspectos medioambientales ( $\bar{x}$ GapRSEp- $\left.\mathrm{e}_{3}=-1.11\right)$ y, por último, la vinculada a donaciones económicas $\left(\bar{x}\right.$ GapRSEpe $\left._{1}=-0.97\right)$.

Estos hallazgos sirven para ilustrar, con apoyo de la Figura 2 , las ventajas que supone emplear el paradigma de la DE para la medición de la RSE en comparación con la forma tradicional basada exclusivamente en las percepciones. En primer lugar destaca que, al ser necesario medir las expectativas de los stakeholders para la determinación de las brechas, se posibilita conocer, tanto la importancia relativa atribuida por los sujetos a cada una de las cuestiones de RSE, como los límites mínimos deseables que los decisores de la firma han de procurar alcanzar para satisfacerlos. En segundo lugar, conocer el sentido y la magnitud de cada gap incrementa la sensibilidad de los directivos para jerarquizar el destino de los recursos y esfuerzos de RSE a su cargo y, en tercer lugar, obtienen información valiosa y precisa para dimensionar los costos y oportunidades para alcanzar sus objetivos de RSE.

Figura 2 Gaps RSEp-e

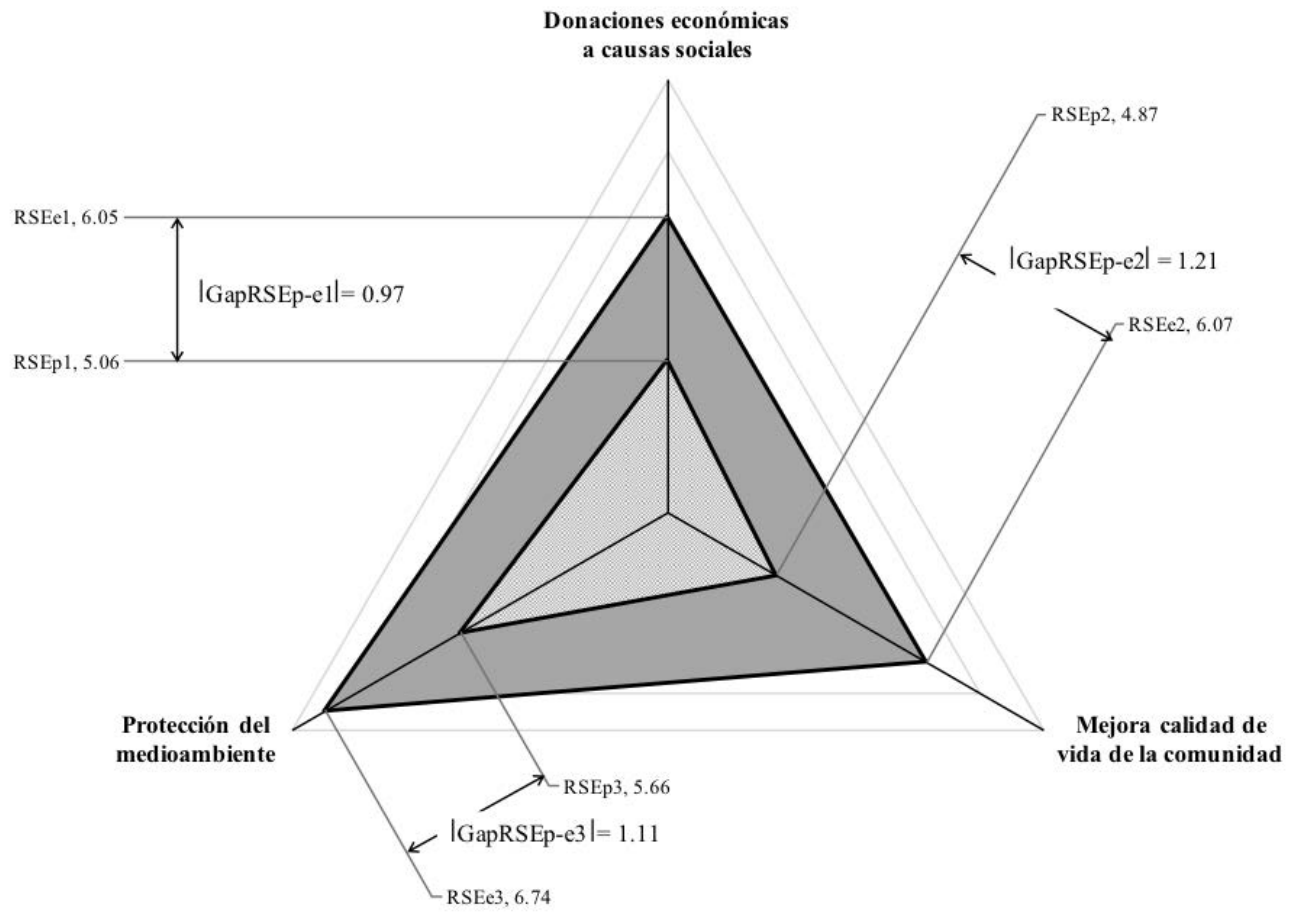


Lo anterior contribuye entonces a que los decisores puedan dirigir sus esfuerzos y recursos de forma más atinada y precisa y, en consecuencia, ayudarles a mejorar aquellos aspectos específicos de RSE que así lo requieran; ya sea que se trate de las propias acciones y programas o de su comunicación efectiva a los distintos públicos.

Por ejemplo, en este caso, el hecho de que las percepciones de los empleados se hayan ubicado por debajo de sus expectativas puede atribuirse a diversos factores exógenos y endógenos de la organización; por un lado, es posible que las demandas sociales sean poco realistas y/o desproporcionadas a la capacidad de la firma para atenderlas y por otro lado, a que los esfuerzos de RSE realizados por la firma hayan sido insuficientes, que el ajuste de los programas, actividades y campañas no sea el adecuado para su giro y/o que estos no hayan sido comunicados de forma efectiva a este grupo de interés en particular, entre otros.

Esto ayuda a destacar que, si bien el modelo de las AC (Brown y Dacin, 1997) ha permitido medir de forma fiable y válida el Gap RSEp-e en este estudio, las conclusiones que es posible extraer a partir de su escala son de carácter demasiado general, lo que refuerza hallazgos previos en torno a sus limitaciones y la necesidad de recurrir a otros paradigmas (Alvarado y Schlesinger, 2008; Alvarado et al., 2017; García de los Salmones et al., 2005) que permitan conocer de manera detallada cuáles aspectos de RSE es necesario ajustar, corregir, mejorar, continuar o suspender para atender los gaps descubiertos.

\subsubsection{Variables consecuentes del Gap RSEp-e}

Los estadísticos descriptivos correspondientes a las variables consecuentes contempladas en esta investigación se presentan en la Tabla 5. Como puede observarse, los valores obtenidos en todos los casos son superiores a 5.5 y oscilan entre $5.63\left(\mathrm{IP}_{1}\right)$ y $6.48\left(\mathrm{IO}_{6}\right)$, lo que indica que los empleados participantes dijeron estar identificados con el hotel para el que trabajan, motivados laboralmente y tener intención de continuar laborando para la empresa.
Tabla 5 Estadísticos descriptivos de las variables consecuentes

\begin{tabular}{|c|c|c|c|c|c|}
\hline Variable & Item & $\bar{x}$ & $\Sigma$ & Min. & Max \\
\hline \multirow[t]{7}{*}{ IO } & $\mathrm{IO}_{1}$ & 6.04 & 1.24 & 1 & 7 \\
\hline & $\mathrm{IO}_{2}$ & 6.14 & 1.16 & 3 & 7 \\
\hline & $\mathrm{IO}_{3}$ & 6.22 & 1.04 & 2 & 7 \\
\hline & $\mathrm{IO}_{4}$ & 6.05 & 1.22 & 2 & 7 \\
\hline & $\mathrm{IO}_{5}$ & 6.17 & 1.10 & 2 & 7 \\
\hline & $\mathrm{IO}_{6}$ & 6.48 & 0.88 & 3 & 7 \\
\hline & $\mathrm{IO}_{7}$ & 5.96 & 1.41 & 1 & 7 \\
\hline \multirow[t]{4}{*}{ ML } & $\mathrm{ML}_{1}$ & 6.08 & 1.19 & 1 & 7 \\
\hline & $\mathrm{ML}_{2}$ & 6.10 & 1.22 & 1 & 7 \\
\hline & $\mathrm{ML}_{3}$ & 6.11 & 1.20 & 1 & 7 \\
\hline & $\mathrm{ML}_{4}$ & 6.07 & 1.25 & 1 & 7 \\
\hline \multirow[t]{3}{*}{ IP } & $\mathrm{IP}_{1}$ & 5.63 & 1.59 & 1 & 7 \\
\hline & $\mathrm{IP}_{2}$ & 6.01 & 1.35 & 1 & 7 \\
\hline & $\mathrm{IP}_{3}$ & 5.95 & 1.33 & 1 & 7 \\
\hline
\end{tabular}




\subsection{Contrastación de hipótesis y del modelo en su conjunto}

Una vez verificadas la fiabilidad y validez del modelo de medida y descritos los estadísticos básicos de las variables estudiadas, se procede a contrastar las hipótesis planteadas y el modelo teórico propuesto en su conjunto mediante el modelo de ecuaciones estructurales (MEC) correspondiente. Los resultados del análisis se muestran en la Tabla 6 y apuntan a un buen ajuste global $\left(\mathrm{S}-\mathrm{B}_{X}^{2}(115 \mathrm{gl})=161.65, \mathrm{p}<0.01\right.$; $\mathrm{NFI}=0.884 ; \quad \mathrm{NNFI}=0.956 ; \quad \mathrm{CFI}=0.963 ; \quad \mathrm{IFI}=0.964$; $\mathrm{MFI}=0.890 ; \mathrm{RMSEA}=0.045)$.

Tabla 6 Resultados del MEC



$* \mathrm{p}<0.05, * * \mathrm{p}<0.01$

Como puede apreciarse, los resultados de los análisis sugieren que las relaciones estructurales propuestas en las cuatro hipótesis son estadísticamente significativas $\left(\mathrm{H}_{1}, \mathrm{H}_{2}\right.$ y $\mathrm{H}_{3}$ para $\mathrm{p}<0.01 \mathrm{y} \mathrm{H}_{4}$ para $\left.\mathrm{p}<0.05\right)$ y tienen el sentido previsto $(+)$ por lo que no pueden ser rechazadas y, en consecuencia, se aceptan. Estos hallazgos están en consonancia con los postulados de Swaen (2003) y Alvarado et al. (2010) en relación a que el PDE (Anderson, 1973) es útil para medir y explicar, a partir de las nociones de las percepciones, expectativas y discrepancias entre ambas (Grönroos, 1982; Lehtinen y Lehtinen, 1982; Parasuraman et al., 1988), la RSE y sus influencias sobre variables específicas del comportamiento de distintos stakeholders. Consecuentemente, dan soporte y validan de forma externa los reportados en el trabajo que la originó y lo hacen, además, a partir de un contexto geográfico y social distintos, lo que contribuye a ampliar la generalización de los resultados. El modelo final estimado descrito en la Tabla 6 se presenta en la Figura 3, incluyendo los ítems de cada factor y sus cargas estandarizadas, para asistir en su contrastación general.

\subsubsection{Análisis de los efectos directos entre las variables . antecedentes y sus consecuentes}

La aceptación de H1 permite confirmar por primera vez, que el gap entre las percepciones y las expectativas de la RSE de los empleados influye de forma directa y significativa $(\mathrm{p}<0.01)$ sobre su identificación con la empresa y que lo hace en una magnitud importante ( $\lambda$ Gap RSEp-e $\rightarrow \mathrm{IO}=0.361$ ). Esta contribución confirma la idea de que las actividades y programas de RSE refuerzan su compromiso afectivo con la empresa (Brammer et al., 2007; Kim et al., 2010; Serra et al., 2017) y respalda la noción de que los empleados pueden satisfacer parte de sus necesidades de definición personal mediante la incorporación de rasgos de la personalidad de la organización en la que se desenvuelven profesionalmente para enriquecer el concepto que tienen de sí mismos (Bhattacharya et al., 2008; Dutton et al., 1994; Kim et al., 2010). 
Figura 3 Modelo final.

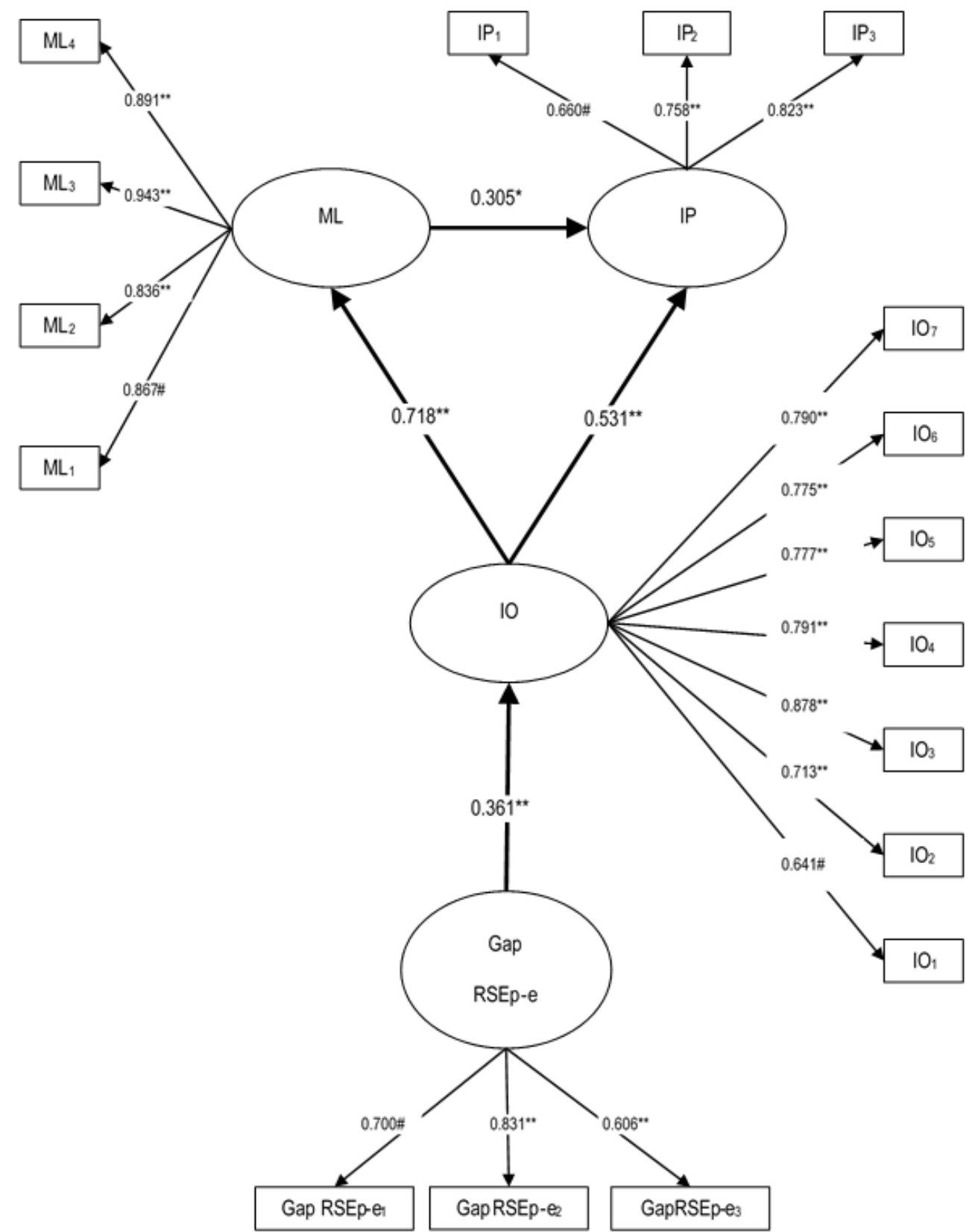

$* p<0.01$

\# Parámetro inicial fijado a 1 para sobre identificación del modelo 
La contrastación y consecuente aceptación de $\mathrm{H}_{2}$, indica que la IO basada en el GAP RSEp-e tiene un efecto directo positivo, significativo $(\mathrm{p}<0.01)$ y de gran impacto en la motivación de los trabajadores $\left(\lambda \mathrm{IO}{ }_{-} \rightarrow \mathrm{ML}=0.718\right)$. Algo similar sucede con el resultado obtenido de la contrastación de H3, cuya aceptación supone demostrar que la ML resultante del Gap RSEp-e influye de forma directa, positiva, significativa $(\mathrm{p}<0.01)$ y relevante $(\lambda \mathrm{IO} \rightarrow \mathrm{IP}=0.531)$ sobre la intención de los trabajadores de continuar laborando para la empresa. En lo tocante a H4, se considera probado que, desde la PDE, la ML derivada de la disconfirmación de las percepciones y expectativas de la RSE influye directa, significativa y positivamente en la intención de los empleados de continuar prestando sus servicios para la compañía en la que trabajan $(\mathrm{p}<0.05, \lambda \mathrm{ML} \rightarrow \mathrm{IP}=0.305)$.

Estos tres hallazgos constituyen igual número de contribuciones al conocimiento de la RSE, pues hasta donde se sabe, nunca antes habían sido estudiados y confirmados empíricamente desde la perspectiva de la PDE ni en este ámbito de estudio.

\subsubsection{Análisis de los efectos indirectos y totales del Gap RSEp-e sobre las variables consecuentes}

Adicionalmente, estos resultados permiten descubrir los mecanismos que explican los efectos indirectos y totales ejercidos por el Gap RSEp-e tanto sobre la ML a través de la IO, como sobre la IP a través de ambas, y sus magnitudes, tal como se expone a continuación. En lo correspondiente a los efectos totales ejercidos por el Gap RSEp-e sobre la ML estos son $\boldsymbol{\lambda}_{\text {TотALES }}$ Gap_RSEp-e $\rightarrow \mathrm{ML}=0.259$, para $\mathrm{p}<0.01$, los cuales se determinaron según la Fórmula [1]:



Este resultado, además de consolidar lo señalado en párrafos anteriores en relación a que la RSE refuerza el compromiso afectivo de los empleados con la empresa (Brammer et al., 2007; Kim et al., 2010; Serra et al., 2017) y que concurre al enriquecimiento de su autoconcepto (Bhattacharya et al., 2008; Dutton et al., 1994; Kim et al., 2010), soporta los postulados de Scott y Lane (2000) en el sentido de que la conexión psicológica de los individuos con la organización para la que laboran favorece el desarrollo de conductas deseables que potencian su rendimiento y, consecuentemente, el de la firma. De forma análoga, en lo referente a los efectos indirectos y totales del Gap RSEp-e sobre la IP se tienen: en primer lugar, los ejercidos a través de la mediación de la IO, dados por la Fórmula [2]:

$\lambda_{\text {INDIRECTOSGap RSE }} \rightarrow \boldsymbol{\rightarrow} I O \rightarrow I P=\left(\lambda G_{\text {Gap RSE }} \rightarrow \boldsymbol{\rightarrow} I O\right)(\lambda I O \rightarrow I P)$ y cuyo valor asciende a: $\lambda_{\text {INDIRECTos }}$ Gap RSEp-e $\rightarrow$ IO $\rightarrow$ $\mathrm{IP}=0.192(\mathrm{p}<0.01)$; $\mathrm{y}$ en segundo lugar, su influencia indirecta a través de la mediación de la IO y la ML, como se expone en la Fórmula [3]:

$$
\begin{aligned}
\lambda_{\text {INDIRECTOS Gap RSE }} \rightarrow \mathrm{\rightarrow} I O & \rightarrow M L \rightarrow I P= \\
\left(\lambda G a p R S E_{p-e}\right. & \rightarrow I O)(\lambda I O \rightarrow M L)(\lambda M L \rightarrow I P)
\end{aligned}
$$

lo que da como resultado $\lambda_{\text {INDIRECTos }}$ Gap RSEp-e $\rightarrow$ IO $\rightarrow$ $\mathrm{ML} \rightarrow \mathrm{IP}=0.079$ para $\mathrm{p}<0.05$. Consecuentemente, los efectos totales ejercidos por el Gap RSEp-e sobre la IP están dados por la suma de los dos anteriores, según se expresa en [4]:

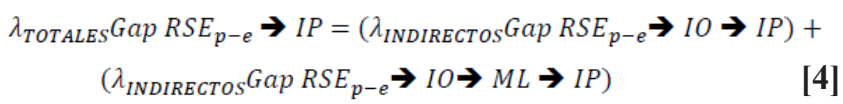

y que son $\lambda_{\text {TOTALES }}$ Gap RSEp-e $\rightarrow \mathrm{IP}=0.271$ para $\mathrm{p}<0.05$. Este hallazgo soporta, además de los aspectos teóricos expresados en los párrafos precedentes (v.g. Bhattacharya et al., 2008; Brammer et al., 2007; Dutton et al., 1994; Kim et al., 2010; Scott y Lane, 2000 y Serra et al., 2017), la idea de Sager y colaboradores (2014) respecto a que la ML es un factor precursor de la IP de los trabajadores en su organización y lo hace, además desde las perspectivas de la RSE, aquí operacionalizada desde el PDE.

\section{Conclusiones, limitaciones y líneas de investigación futura}

Como parte de una línea de investigación mayor sobre RSE, se diseñó y llevó a cabo un estudio cuantitativo de naturaleza confirmatoria que permitió alcanzar tres objetivos: i) se profundizó en la investigación de las brechas entre la RSEp y la RSEe por los empleados de empresas de servicios y ii) en el estudio de sus influencias sobre variables consecuentes relativas a su comportamiento; $y$, por otra parte iii) se validaron externamente hallazgos previos en torno a algunas limitaciones del modelo de las asociaciones corporativas (AC) para la medición y explicación de la RSE.

Los resultados permitieron confirmar empíricamente y documentar por vez primera las influencias directas, indirectas y totales ejercidas por las discrepancias entre las percepciones y expectativas de los trabajadores acerca de la RSE de firmas dedicadas a la prestación de servicios de alojamiento turístico sobre su identificación con la organización, motivación e intención de permanencia laboral, y con ello, descubrir mecanismos mediante los cuales se producen dichos efectos. Consiguientemente fue posible identificar también que las magnitudes de los efectos de los gaps RSEp-e sobre esas variables fueron considerables, lo que apunta a la importancia relativa que este grupo de stakeholders atribuye a las iniciativas, programas y acciones de RSE llevadas a cabo por la organización a la que pertenecen. 
Otras contribuciones de esta investigación se relacionan con la validación externa de los postulados de Alvarado et al. (2010) sobre la utilidad del paradigma de la disconfirmación de expectativas como un instrumento valioso para la medición de la RSE y sus influencias en el comportamiento de los stakeholders. Así, destaca que su uso posibilita conocer las expectativas de RSE y el peso relativo de cada uno de sus aspectos para los empleados; incrementa la sensibilidad de los tomadores de decisiones para ponderar recursos y esfuerzos dedicados a la RSE de la firma y; genera información valiosa para el dimensionamiento de los costos y oportunidades para cerrar las brechas RSEp-e y satisfacer, en consecuencia, a sus colaboradores internos.

Ahora bien, la validación externa realizada refuerza también las ideas en torno a las limitaciones del modelo de las AC previamente acusadas en la literatura. Esto es así, pues aún cuando la escala empleada en esta investigación para medir el GapRSEp-e demostró ser fiable y contar con validez aparente, convergente y discriminante, los contenidos de sus ítems son demasiado generales; por tanto, parece insuficiente para identificar específicamente cuáles son los aspectos y actividades concretos de RSE en los cuales los empleados esperan que sus compañías se involucren. Luego, se mantiene abierta la oportunidad de realizar estudios complementarios que aborden la medición de la RSEp y RSEe y sus gaps desde otras perspectivas teóricas, tales como las ofrecidas por el modelo de Carroll o el paradigma del desarrollo sostenible.

Este trabajo no está exento de limitaciones que abren nuevas oportunidades de investigación y que indican que la generalización de sus resultados ha de hacerse con prudencia. Además de la señalada en el párrafo precedente, se tiene que el estudio aborda únicamente el subsector de servicios de alojamiento turístico y que aún es necesario comprobar empíricamente si el modelo de estudio basado en el Gap RSEp-e es, además de útil, realmente más poderoso (o no) que los instrumentos que lo originan, lo que se propone realizar inicialmente mediante el análisis de modelos competitivos resultantes de investigaciones basadas en los mismos contextos, ámbitos y grupo de stakeholders pero diferenciados por los supuestos de sus aproximaciones teóricas.

No obstante, se considera que los hallazgos de este estudio contribuyen a llenar los vacíos existentes en el conocimiento $\mathrm{y}$ que son insumos valiosos para académicos y profesionales de la administración, especialmente del marketing y la RSE. Los descubrimientos aquí realizados pueden asistir a los tomadores de decisiones para la dirección de sus esfuerzos y recursos de forma más atinada y precisa y, en consecuencia, mejorar aquellos aspectos específicos de RSE que así lo requieran. Sugieren también que aquellos directores y gerentes que atiendan el Gap RSEp-e y sean capaces de desarrollar estrategias de RSE, inteligentes y congruentes, y de afianzarlas en la filosofía y valores de sus empresas de forma tal que superen las expectativas de sus colaboradores, podrán obtener ventajas competitivas sostenibles y hacer frente al imperativo estratégico al que se refieren Serra y colaboradores (2017).

\section{Referencias}

Alvarado, A., Bigné, E., Aldás, J., y Currás, R. (2017). A scale for measuring consumer perceptions of corporate social responsibility following the sustainable development paradigm. Journal of Business Ethics, 140(2), 243262.

Alvarado, A., Bigné, E., Currás, R., y Aldás, J. (2010). Does corporate social responsibility really add value for consumers? En C. Louche, S. Idowu y W. Leal (eds.), Innovative CSR: From Risk Management to Value Creation (pp. 173-195). Sheffield: Greenleaf.

Alvarado, A., y Schlesinger, M. W. (2008). Dimensionalidad de la responsabilidad social empresarial percibida y sus efectos sobre la imagen y la reputación: una aproximación desde el modelo de Carroll. Estudios gerenciales, 24(108), 37-59.

Anderson, E. W., y Sullivan, M. W. (1993). The Antecedents and Consequences of Customer Satisfaction for Firms. Marketing Science, 12(2), 125-143. https://doi. org/10.1287/mksc.12.2.125

Anderson, J. C., y Gerbing, D. W. (1988). Structural equation modeling in practice: A review and recommended two-step approach. Psychological bulletin, 103(3), 411.

Anderson, R. E. (1973). Consumer Dissatisfaction: The Effect of Disconfirmed Expectancy on Perceived Product Performance. Journal of Marketing Research, 10(1), 3844.

Bagozzi, R. P., y Yi, Y. (1988). On the evaluation of structural equation models. Journal of the academy of marketing science, 16(1), 74-94.

Bañuls, A. L., Rodríguez, A. B. R., y Jiménez, M. S. (2007). El capital humano como factor estratégico para la competitividad del sector turístico. Cuadernos de turismo, (19), 47-69.

Barnard, C. I. (1938). The functions of the executive. Cambridge, Mass: Harvard University Press.

Bentler, P.M. (1985-2017). EQS (Versión 6.3). Temple City, CA: Multivariate Software, Inc.

Bergami, M., y Bagozzi, R. P. (2000). Self-categorization, affective commitment and group self-esteem as distinct aspects of social identity in the organization. British Journal of Social Psychology, 39(4), 555-577. 
Bernstein, I. H., y Nunnally, J. C. (1994). Psychometric theory. New York: McGraw-Hill.

Bhattacharya, C. B., Sen, S., y Korschun, D. (2008). Using Corporate Social Responsibility to Win the War for Talent. MIT Sloan Management Review, 49(2), 37-44.

Bhattacherjee, A. (2001). Understanding Information Systems Continuance: An Expectation Conifrmation Model. Mis Quarterly, 25(3), 351-370.

Bigné Alcañiz, E., Alvarado Herrera, A., Currás Pérez, R., y Rivera Alcami, J. J. (2010). Latest evolution of academic research in corporate social responsibility: an empirical analysis. Social Responsibility Journal, 6(3), 332-344.

Bowen, H. R. (1953). Social responsibilities of the businessman. New York: Harper.

Brammer, S., Millington, A., y Rayton, B. (2007). The contribution of corporate social responsibility to organizational commitment. The International Journal of Human Resource Management, 18(10), 1701-1719.

Brønn, P. S., y Vrioni, A. B. (2001). Corporate social responsibility and cause-related marketing: an overview. International Journal of Advertising, 20(2), 207-222.

Brown, T. J., y Dacin, P. A. (1997). The company and the product: Corporate associations and consumer product responses. Journal of Marketing, 61(1), 68-84.

Carroll, A. B. (1979). A three-dimensional conceptual model of corporate performance. Academy of management review, 4(4), 497-505.

Carroll, A. B. (1999). Corporate social responsibility: Evolution of a definitional construct. Business \& society, $38(3), 268-295$

Chandon, P., Morwitz, V. G., y Reinartz, W. J. (2005). Do intentions really predict behavior? Self-generated validity effects in survey research. Journal of Marketing, 69(2), $1-14$.

Cronin, J. J., Brady, M. K., y Hult, G. T. M. (2000). Assessing the Effects of Quality, Value, and Customer Satisfaction on Consumer Behavioral Intentions in Service Environments. Journal of Retailing, 76(2), 193-218.

Dutton, J. E., Dukerich, J. M., y Harquail, C. V. (1994). Organizational Images and Member Identification. Administrative Science Quarterly, 39, 239-263.

Fornell, C., y Larcker, D. F. (1981). Structural Equation Models with Unobservable Variables and Measurement Error: Algebra and Statistics. Journal of Marketing Research, 18(3), 382-388.
Freeman, R. E., y Reed, D. L. (1983). Stockholders and Stakeholders: A New Perspective on Corporate Governance. California Management Review, 25(3), 88-106. https://doi.org/10.2307/41165018

Friedman, M. (1962). Capitalism and freedom. Chicago, IL: University of Chicago Press.

García de los Salmones, M., Herrero, A., y Rodríguez del Bosque, I. (2005). Influence of corporate social responsibility on loyalty and valuation of services. Journal of business ethics, 61(4), 369-385.

Gorden, W. I., Anderson, C. M., y Bruning, S. D. (1992). Employee perceptions of corporate partnership: An affective-moral quid pro quo. Employee Responsibilities and Rights Journal, 5(1), 75-85. https://doi.org/10.1007/ $\underline{\text { BF01407810 }}$

Grönroos, C. (1982). An applied service marketing theory. European journal of marketing, 16(7), 30-41.

Hair, J. F., Black, W. C., Babin, B. J., Anderson, R. E., y Tatham, R. L. (1998). Multivariate Data Analysis. Upper Saddle River, NJ: Prentice hall.

Heugens, P. P. M. A. R., van den Bosch, F. A. J., y van Riel, C. B. M. (2002). Stakeholder Integration: Building Mutually Enforcing Relationships. Business \& Society, 41(1), 36-60. https://doi.org/10.1177/000765030204100104

https://doi.org/10.1080/02650487.2001.11104887

Instituto Nacional de Estadística y Geografía. (2016). Anuario estadístico y geográfico de Quintana Roo 2016. México: INEGI

Janssen, C., Sen, S., y Bhattacharya, C. B. (2015). Corporate crises in the age of corporate social responsibility. Business Horizons, 58(2), 183-192.

Kandasamy, I., y Ancheri, S. (2009). Hotel employees' expectations of QWL: A qualitative study. International Journal of Hospitality Management, 28(3), 328-337. https://doi.org/10.1016/j.ijhm.2008.11.003

Kim, H. R., Lee, M., Lee, H. T., y Kim, N. M. (2010). Corporate social responsibility and employee-company identification. Journal of Business Ethics, 95(4), 557-569.

Kim, J., Song, H.-J., Lee, C.-K., y Lee, J.-Y. (2017). The impact of four CSR dimensions on a gaming company's image and customers' revisit intentions. International Journal of Hospitality Management, 61, 73-81. https:// doi.org/https://doi.org/10.1016/i.ijhm.2016.11.005 
Kline, R. B. (2015). Principles and practice of structural equation modeling. Guilford publications.

Lehtinen, U., y Lehtinen, J. R. (1982). Service quality: a study of quality dimensions. Helsinky: Service Management Institute.

Luque-González, A., Hernández-Zubizarreta, J., y de Pablos-Heredro, C. (2016). Oportunidades dentro de los procesos de mundialización textil internacional y relación con la RSE a través de un analisis DELPHI: ética o estética. Dirección y Organización, (59), 32-48.

Mael, F., y Ashforth, B. E. (1992). Alumni and their alma mater: A partial test of the reformulated model of organizational identification. Journal of organizational Behavior, 13(2), 103-123.

Maignan, I. (2001). Consumers' perceptions of corporate social responsibilities: A cross-cultural comparison. Journal of business ethics, 30(1), 57-72.

Maignan, I., y Ferrell, O. C. (2003). Nature of corporate responsibilities: Perspectives from American, French, and German consumers. Journal of Business research, 56(1), 55-67.

Markovic, S., Iglesias, O., Singh, J. J., y Sierra, V. (2018). How does the Perceived Ethicality of Corporate Services Brands Influence Loyalty and Positive Word-ofMouth? Analyzing the Roles of Empathy, Affective Commitment, and Perceived Quality. Journal of Business Ethics, 148(4), 721-740. https://doi.org/10.1007/ s10551-015-2985-6

Martínez, P., y Rodríguez del Bosque, I. (2016). The role of consumer identification with the company on the effects of corporate social responsibility associations on consumer behavior, Revista de Análisis Turístico, 22, 12-27.

McWilliams, A., Siegel, D., y Wright, P. (2006). Corporate Social Responsibility: Strategic Implications*. Journal of Management Studies, 43(1), 1-18. https://doi. org/10.1111/j.1467-6486.2006.00580.x

Mejías, A., García, J., Prado, J. C., Fernández, A., y Comesaña, J. A. (2011). Modelo para la aplicación de la Responsabilidad Social Corporativa en la Gestión de la Cadena de Suministro. Dirección y Organización, (45), 20-31.

Mowday, R. T., Steers, R. M., y Porter, L. W. (1979). The measurement of organizational commitment. Journal of vocational behavior, 14(2), 224-247.
Oliver, R. (1980). A Cognitive Model of the Antecedents and Consequences of Satisfaction Decisions. Journal of Marketing Research, 17(4), 460-469.

Organización Internacional del Trabajo (OIT). (2001). El desarrollo de los recursos humanos, el empleo y la mundialización en el sector de la hotelería, la restauración y el turismo. Informe para el debate de la Reunión tripartita sobre el desarrollo de los recursos humanos, el empleo y la mundialización en el sector de la hotelería, la restauración y el turismo. Ginebra, Suiza.

Parasuraman, A., Zeithaml, V. A., y Berry, L. L. (1985). A Conceptual Model of Service Quality and Its Implications for Future Research. Research Paper, 49(4), 41-50. https://doi.org/10.1016/S0148-2963(99)00084-3

Parasuraman, A., Zeithaml, V. A., y Berry, L. L. (1988). SERVQUAL: A Multiple-Item Scale for Measuring Consumer Perceptions of Service Quality. Journal of Retailing, 64(1), 12-40.

Sager, J. K., Naletelich, K., y Dubinsky, A. J. (2014). Examination of Selected Precursors and Outcomes of Sales Manager Behaviors. International Business Research, 7(9), 91.

Satorra, A., y Bentler, P. M. (1988, August). Scaling corrections for chi-square statistics in covariance structure analysis. In Proceedings of the American Statistical Association (Vol. 1, pp. 308-313).

Scott, S. G., y Lane, V. R. (2000). A stakeholder approach to organizational identity. Academy of Management review, 25(1), 43-62.

Serra-Cantallops, A., Peña-Miranda, D. D., Ramón-Cardona, J., y Martorell-Cunill, O. (2017). Progress in Research on CSR and the Hotel Industry (2006-2015). Cornell Hospitality Quarterly, (OnlineFirst, July 10, 2017), 1938965517719267.

Stoney, C., y Winstanley, D. (2001). Stakeholding: Confusion or Utopia? Mapping the Conceptual Terrain. Journal of Management Studies, 38(5), 603-626. https://doi. org/10.1111/1467-6486.00251

Swaen, V. (2003). Consumers' Perceptions, Evaluations and Reactions to CSR Activities (IESEG working paper 2003-mar-7; Lille, France: IESEG).

Vintró, C., y Fortuny, J. (2010). Estudio empírico de las prácticas de Responsabilidad Social Corporativa en la minería de áridos en Cataluña Empirical study of CSR practices in the aggregate mining in Catalonia. Dirección y Organización, (42), 16-23. 
Dr. Alejandro Alvarado Herrera. / Dirección y Organización 67 (2019) 5-21

Anexo 1 Operacionalización de las variables.

\begin{tabular}{|c|c|c|}
\hline Variable & Ítem & Contenido \\
\hline \multirow[t]{4}{*}{ RSEp } & & En mi opinión, el hotel [marca de la cadena] realmente intenta... \\
\hline & $\operatorname{RSEp}_{1}$ & ... hacer donaciones económicas a causas sociales \\
\hline & $\mathrm{RSEp}_{2}$ & ... mejorar la calidad de vida de la comunidad local \\
\hline & $\mathrm{RSEp}_{3}$ & ... proteger el medioambiente \\
\hline \multirow[t]{4}{*}{ RSEe } & & En mi opinión, el hotel [marca de la cadena] debería... \\
\hline & $\mathrm{RSEe}_{1}$ & ... hacer donaciones económicas a causas sociales \\
\hline & $\mathrm{RSEe}_{2}$ & ... mejorar la calidad de vida de la comunidad local \\
\hline & $\mathrm{RSEe}_{3}$ & ... proteger el medioambiente \\
\hline
\end{tabular}

IO

$\mathrm{IO}_{1} \quad$ Pienso que mi empleo en el Hotel [marca de la cadena] es una gran parte de quién soy

$\mathrm{IO}_{2} \quad$ Me considero una persona [marca de la cadena]

$\mathrm{IO}_{3} \quad$ Lo que el hotel [marca de la cadena] significa, es importante para mí

$\mathrm{IO}_{4} \quad$ Comparto los objetivos del [marca de la cadena]

$\mathrm{IO}_{5} \quad$ Comparto los valores del hotel [marca de la cadena]

$\mathrm{IO}_{6} \quad$ Mi empleo en el hotel [marca de la cadena] es importante para mí

$\mathrm{IO}_{7} \quad$ Siento fuertes lazos con el hotel [marca de la cadena]

ML $\quad \mathrm{ML}_{1} \quad$ Sin lugar a dudas, mi empleo en el hotel [marca de la cadena] es muy motivante para mi

$\mathrm{ML}_{2} \quad$ La motivación con que desempeño mi trabajo en el hotel [marca de la cadena] es muy grande

$\mathrm{ML}_{3} \quad$ Trabajar en el hotel [marca de la cadena] es realmente motivante para mi

$\mathrm{ML}_{4} \quad$ Mi trabajo en el hotel [marca de la cadena] siempre es altamente motivante para mi

IP $\quad \mathrm{IP}_{1} \quad$ Si por alguna circunstancia tuviera que cambiar mi empleo, es prácticamente seguro que trabajaría en otro hotel [marca de la cadena]

$\mathrm{IP}_{2} \quad$ En lo que a mí respecta, probablemente seré empleado del hotel [marca de la cadena] por largo tiempo

$\mathrm{IP}_{3} \quad \mathrm{Si}$ me corresponde tomar la decisión, la posibilidad de que trabaje para un hotel [marca de la cadena] en el fututo es muy alta

Fuente: Elaboración propia basada Brown y Dacin (1997), Alvarado et al. (2010), Escalas y Bettman (2003) y Chandon et al. (2005) 